\title{
STELLAR ARCHAEOLOGY: A KECK PILOT PROGRAM ON EXTREMELY METAL-POOR STARS FROM THE HAMBURG/ESO SURVEY. I. STELLAR PARAMETERS ${ }^{1}$
}

\author{
Judith G. Cohen, ${ }^{2}$ Norbert Christlieb, ${ }^{3}$ Timothy C. Beers, ${ }^{4}$ Raffaele Gratton, ${ }^{5}$ and Eugenio Carretta 5 \\ Received 2001 October 1; accepted 2002 March 26
}

\begin{abstract}
In this series of two papers, we present a high-dispersion spectroscopic analysis of eight candidate extremely metal-poor stars selected from the Hamburg/ESO Survey (HES) and of six additional very metalpoor stars. We demonstrate that with suitable vetting using moderate-resolution spectra, the yield of this survey for stars with $[\mathrm{Fe} / \mathrm{H}] \leq-3.0$ dex is very high; three out of the eight stars observed thus far at high resolution from the HES are actually that metal-poor, three more have $[\mathrm{Fe} / \mathrm{H}] \leq-2.8$ dex, and the remainder are only slightly more metal-rich. In preparation for a large-scale effort to mine the HES database for such stars about to get under way, we lay out in this paper the basic principles we intend to use to determine in a uniform way the stellar parameters $T_{\text {eff }}, \log g$, and reddening.
\end{abstract}

Key words: Galaxy: evolution — Galaxy: halo — stars: abundances

\section{INTRODUCTION}

The most metal-deficient stars in the Galaxy provide crucial evidence on the early epoch of the formation of our Galaxy, the chemical evolution of the Galaxy, the environment in which various elements were produced, the production of elements in the big bang, the age of the Galaxy, the relationship between the halo field stars and the Galactic globular clusters, etc. We consider only stars with $[\mathrm{Fe} / \mathrm{H}] \leq-3$ dex $(<1 / 1000$ of the solar metallicity) to be extremely metalpoor (EMP) and most useful for discussion of such issues. These EMP stars provide us with an opportunity to study in detail the local equivalent of the high-redshift universe.

The major existing survey for very metal-poor stars is the HK survey (previously referred to as the Preston-Shectman survey), described in detail by Beers, Preston, \& Shectman $(1985,1992)$. This survey produced a list of $\sim 10,000$ candidate metal-poor stars. Extensive follow-up studies, which continue to this date, now include moderate-resolution ( $\Delta \lambda \sim 1-2 \AA$ ) spectroscopy of about 5000 stars and broadand narrowband photometry for some 3000 stars. From this work, one can cull a sample of roughly 1000 stars with $[\mathrm{Fe} / \mathrm{H}] \leq-2.0$ dex, but as summarized by Beers (1999), only roughly 100 are known to be extremely metal-poor. High-dispersion analyses exist for only a few stars with $[\mathrm{Fe} / \mathrm{H}] \leq-3.5 \mathrm{dex}$, and the signal-to-noise ratios of these spectra are, in general, modest $(\sim 30-40)$, a situation that only very recently has been partially remedied for five giants through the work of Norris, Ryan, \& Beers (2001), and for the most metal-poor dwarf presently known, CS 22876-032 (Norris, Beers, \& Ryan 2000).

\footnotetext{
${ }^{1}$ Based in large part on observations obtained at the W. M. Keck Observatory, which is operated jointly by the California Institute of Technology, the University of California, and NASA.

2 Palomar Observatory, Mail Stop 105-24, California Institute of Technology, 1201 East California Boulevard, Pasadena, CA 91125; jlc@ astro.caltech.edu.

${ }^{3}$ Hamburger Sternwarte, Gojenbergsweg 112, D-21029 Hamburg, Germany; nchristlieb@hs.uni-hamburg.de.

${ }^{4}$ Department of Physics and Astronomy, Michigan State University, East Lansing, MI 48824-1116; beers@pa.msu.edu.

${ }^{5}$ Osservatorio Astronomico di Padova, Instituto Nazionale di Astrofisica, vicolo dell'Osservatorio 5, I-35122, Padova, Italy, carretta@ pd.astro.it, gratton@pd.astro.it.
}

McWilliam (1997) reviews the key results from abundance analyses for the most metal-poor stars known from the $\mathrm{HK}$ survey. The elements $\mathrm{Al}, \mathrm{Sr}, \mathrm{Ba}, \mathrm{Cr}, \mathrm{Mn}$, and $\mathrm{Co}$ show a sudden change in the slope of $[\mathrm{X} / \mathrm{Fe}]$ versus $[\mathrm{Fe} / \mathrm{H}]$ near $[\mathrm{Fe} / \mathrm{H}]=-2.4$ dex. Other elements, including $\mathrm{Ba}$ and $\mathrm{Sr}$, appear to show considerable scatter from star to star at very low $[\mathrm{Fe} / \mathrm{H}]$, perhaps indicating that the yield from individual or at most a few supernova events produced the metals seen in these EMP stars. However, the total sample of stars involved is 33 in the McWilliam et al. (1995) study and 19 in Ryan, Norris, \& Beers (1996), with some overlap between these two samples, and many of these do not fit the strict definition of EMP stars adopted here.

The large scatter in the element-to-element abundance ratios observed among EMP stars might reveal a wealth of data for nucleosynthesis. However, a proper understanding of this scatter requires large samples. Furthermore, interesting new types of stars, such as those extremely rich in $r$-process elements (useful for nucleocosmochronology) and extreme $\mathrm{CH}$ stars (used to study the $s$-process), have been found based on the HK survey, but the number known in either case is very small and urgently needs augmentation.

The Hamburg/ESO Survey (HES) is an objective-prism survey primarily targeting bright quasars (Wisotzki et al. 1996, 2000). However, because its spectral resolution is typically $15 \AA$ FWHM at $\mathrm{H} \gamma$, it is also possible to efficiently select a variety of interesting stellar objects in the HES (Christlieb 2000; Christlieb et al. 2001a, 2001b), among them EMP stars. The HES is based on automated scans of objective-prism plates. With a nominal area of $9575 \mathrm{deg}^{2}$, it covers the entire southern extragalactic sky $\left(|b| \geq 30^{\circ}\right.$ and $\left.\delta<+2^{\circ} .5\right)$. The HES limiting magnitude for metal-poor stars is $B \sim 17.5$. The survey is now completed, and the HES database contains digitized objective-prism spectra for about 4 million stars.

A comparison of the EMP stars likely to be found with this survey as compared with the HK survey is given in Christlieb \& Beers (2000). The HES has a number of crucial advantages over the HK survey that should lead to a major increase in the samples of EMP stars and, hence, with suitable follow-up observations, in our knowledge of their properties. The advantages of the HES include a deeper magnitude limit, broader spectral coverage, and automated 
selection from digitized scans of the plates. The latter ensures a selection of EMP stars that can be independent of stellar effective temperature.

The existence of a new list of candidates for EMP stars with $[\mathrm{Fe} / \mathrm{H}]<-3$ dex selected in an automated and unbiased manner from the HES, coupled with the very large collection area and efficient high-resolution echelle spectrographs of 8-10 m telescopes such as Keck plus HIRES or the VLT plus UVES, offers the possibility of a very large increase in the number of EMP stars known and in our understanding of their properties. The present pair of papers (this paper and Paper II, Carretta et al. 2002) describes our very successful pilot project with HIRES at the Keck Observatory in the fall of 2000 to determine the effective yield of the HES for EMP stars through high-dispersion abundance analyses of a sample of stars selected from the HES. Our work complements and extends that of Depagne et al. (2000), who analyzed two stars from the HES as part of the UVES science verification at the VLT.

This success has provided the stimulus for the creation of a long-term large-scale international effort to mine the HES for EMP stars (the " $0 \mathrm{Z}$ Project"), which is now getting under way and which will complement the efforts of the large ESO-VLT program led by R. Cayrel (see, e.g., Cayrel et al. 2001) dedicated to studying the HK survey stars themselves in more detail. In this pair of papers, we strive to lay down at least some of the procedures that we will follow in our long-term effort, the goal of which is to dramatically increase the sample of known and well-studied EMP stars. This first paper is dedicated to determination of the stellar parameters.

\section{THE SAMPLE OF STARS OBSERVED}

Selection of EMP stars in the HES is carried out via automatic spectral classification, using classical statistical methods (Christlieb 2000). ${ }^{6}$ As described in Christlieb et al. (2001b), $B-V$ colors can be estimated directly from the digital HES spectra with an accuracy of $\sim 0.1 \mathrm{mag}$, so these samples can be selected not only on the basis of spectroscopic criteria, but also with restrictions on $B-V$ color.

The principal spectroscopic criterion used for sample selection for EMP stars is the same as that used by the HK project, the absence or weakness of the $3933 \AA$ line of Ca II. A visual check of the HES spectrum is then made to eliminate the small fraction of spurious objects (plate defects, misidentifications, etc.) that pass the automatic selection criteria. The details of this procedure will be discussed elsewhere (Christlieb et al. 2002).

The results from follow-up observations of several hundred metal-poor candidates from the HES will similarly be described elsewhere (Christlieb et al. 2002). Here we only note that in the HES a very high selection efficiency for metal-poor stars has been achieved: $\sim 60 \%$ of the candidate EMP turnoff stars observed at moderate resolution were confirmed to have $[\mathrm{Fe} / \mathrm{H}]<-2.0$ dex, compared with $\sim 30 \%$ in the HK survey (Beers 2000). For the cooler giants, having stronger $\mathrm{Ca} \mathrm{K}$ lines at a given metallicity than turnoff stars, we expect the selection efficiency to be even higher as a result of the still detectable $\mathrm{Ca} \mathrm{K}$ line at very low metallicities. This is currently under investigation.

${ }^{6}$ See http://www.sub.uni-hamburg.de/disse/209/ncdiss.html.
The present sample was selected from the HES database to have $0.3<B-V<0.5$ to focus on main-sequence turnoff stars. The pool of candidates in the turnoff region consists of those stars whose line strengths are below the limit of detection for the HES. This limit corresponds to a metallicity that exceeds the upper limit adopted here for EMP stars, and hence the initial samples are dominated by stars that are very metal-poor but more metal-rich than those we seek. Thus, to make the best use of the limited observing time available on the largest telescopes, these candidates from the HES database must first be verified through moderateresolution $(\sim 1-2 \AA)$ follow-up spectroscopy at $4 \mathrm{~m}$ class telescopes. This procedure selects the genuine EMP stars from the much more numerous stars of slightly higher metallicity — of interest in their own right, but not relevant for our present study. It is the overall efficiency of this multistage selection process for isolating genuine EMP stars that we seek to establish with our pilot project.

\section{HIRES OBSERVATIONS}

Once the list of vetted candidates for EMP candidates from the HES database has been created, observations at high dispersion for a full-scale abundance analysis can be undertaken.

The sample of stars studied here includes seven previously vetted EMP main-sequence turnoff candidates and one giant candidate from the HES. Their coordinates are given in Table 1. One of these turned out to be a rediscovery of a star from the HK survey (HE 2344-2800 = CS 22966-048). For comparison and calibration, three bright, well-studied, very metal-poor stars were observed, two of which (G139-8 and $\mathrm{BD}+3^{\circ} 740$ ) are known to be lithium deficient from the work of Norris et al. (1997) and Ryan, Norris, \& Beers (1999). Three candidate EMP stars from the HK survey were also included. Observations were carried out with the HIRES spectrograph Vogt et al. (1994) at Keck I on two nights in 2000 September. A spectral resolution of 45,000 was achieved using a 0 ". 86 -wide slit projecting to 3 pixels in the HIRES focal-plane CCD detector. The spectra cover the region from 3870 to $5400 \AA$ with essentially no gaps. Each exposure for the HES stars was broken up into $1200 \mathrm{~s}$ segments. The spectra were exposed until a signal-to-noise ratio $(\mathrm{S} / \mathrm{N})$ of 100 per spectral resolution element in the continuum at $4500 \AA$ was achieved. This $\mathrm{S} / \mathrm{N}$ calculation utilizes only Poisson statistics, ignoring issues of cosmic-ray removal, night-sky subtraction, flattening, etc. The observations were carried out with the instrument rotator fixed in the vertical position with respect to the horizon (i.e., at the parallactic angle). As suitable candidates are quite far apart on the sky compared with the $11^{\prime \prime}$-long slit, only a single object can be observed at once.

The exposure times and $\mathrm{S} / \mathrm{N}$ per spectral resolution element in the continuum are given in Table 2. (The gain setting of the HIRES CCD detector is $2.4 e^{-} \mathrm{ADU}^{-1}$; to reach the desired $\mathrm{S} / \mathrm{N}$ of 100 per spectral resolution element requires $1390 \mathrm{ADU}$ pixel $^{-1}$.) These spectra were taken during a period when the Keck I mirror segments had not been aluminized for more than 2 years, and the overall throughput at these blue wavelengths needed improvement.

The spectra from both nights were reduced using the suite of routines for analyzing echelle spectra written by McCarthy (1988) within the Figaro image processing package (Shortridge 1993). The stellar data are flat-fielded with 
TABLE 1

CoOrdinates For the HES Stars In the HIRES SAMPle

\begin{tabular}{|c|c|c|c|}
\hline Name & $\begin{array}{c}V \\
(\mathrm{mag})\end{array}$ & $\begin{array}{c}\text { R.A. } \\
(\mathrm{J} 2000.0)\end{array}$ & $\begin{array}{c}\text { Decl. } \\
(\mathrm{J} 2000.0)\end{array}$ \\
\hline HE $2133-1426 \ldots$ & 15.484 & 213607.2 & -141236 \\
\hline HE $2344-2800^{\mathrm{a}}$. & 14.856 & 234644.4 & -274410 \\
\hline HE $0024-2523^{b} .$. & 14.913 & 002727.6 & -250626 \\
\hline HE $0130-2303 \ldots \ldots \ldots$ & 14.758 & 013318.2 & -224836 \\
\hline HE $0132-2439 \ldots \ldots \ldots$. & 14.821 & 013458.8 & -242418 \\
\hline HE $0148-2611 \ldots \ldots . .$. & 14.453 & 015059.5 & -255702 \\
\hline HE $0218-2738^{\mathrm{c}} \ldots \ldots$. & 14.883 & 022104.0 & -272440 \\
\hline HE $0242-0732 \ldots \ldots \ldots$ & 15.793 & 024500.6 & -071942 \\
\hline
\end{tabular}

NotE.- Units of right ascension are hours, minutes, and seconds, and units of declination are degrees, arcminutes, and arcseconds.

${ }^{\text {a }}$ This is a rediscovery of CS 22966-048, originally found in the HK survey.

b This star has easily detectable $\mathrm{CH}$ absorption in the $\mathrm{G}$ band and its lines are resolved.

${ }^{c}$ The HIRES spectra show that this is a double-lined spectroscopic binary.

quartz lamp spectra, thereby removing most of the blaze profile, and the results are normalized to unity by fitting a sixth-order polynomial to line-free regions of the spectrum in each order.

Figures 1 and 2 show sections of the spectra for six of the stars, including the faintest star in our sample, an EMP main-sequence turnoff star with easily detectable absorption in the $\mathrm{G}$ band of $\mathrm{CH}$, and a newly discovered double-lined spectroscopic binary. The first figure shows the order containing the $\mathrm{G}$ band; the strongest two atomic absorption features in this order are the Fe I lines at 4271.8 and 4307.9 $\AA$. In the second figure, the order containing the Sr II line at $4215.5 \AA$ is shown; the strongest absorption line in that order is that of $\mathrm{Ca}$ I at $4226.7 \AA$. To facilitate a visual comparison of the relative strength of the $\mathrm{Sr}$ II line with other absorption lines, the spectra have been shifted in wavelength so as to remove the differences in radial velocity between the stars displayed in the figure. (The values of $v_{r}$ are given in Table 7 below.)

The strong variation of the $\mathrm{Sr}$ II line relative to adjacent features is obvious. This variation is a well-known characteristic of the spectra of metal-poor stars (see, e.g., Ryan, Norris, \& Bessell 1991) and will be discussed at length in Paper II.

HE 0024-2523, in addition to showing the strongest $\mathrm{CH}$ band, also has absorption lines that are resolved, as is clearly shown in Figures 1 and 2. The most likely explanation for this is rotation. If so, this star has a rotational velocity of $\sim 8 \mathrm{~km} \mathrm{~s}^{-1}$. This star will be discussed again in Paper II and also in more detail in Gratton et al. (2002).

\section{STELLAR BROADBAND PHOTOMETRY}

$U B V$ photometry for all the HES stars was obtained at the ESO-Danish $1.54 \mathrm{~m}$ telescope in 2000 November, using DFOSC (Beers et al. 2002). A $1 \sigma$ error of 0.02 mag was assumed for all optical colors. For BS 17447-029, $U B V$ were available from Bonifacio, Monai, \& Beers (2000), but this disagreed badly with the $V$ photometry from AnthonyTwarog et al. (2000). Because of this large discrepancy, $B V$ for this star were reobserved at the $1.5 \mathrm{~m}$ telescope at Palomar Mountain. The result, given in Table 2, was identical to that of Anthony-Twarog et al. Each of the remaining two stars from the HK survey had two independent sets of optical photometry from either Preston, Shectman, \& Beers (1991), Anthony-Twarog et al. (2000), or McWilliam et al. (1995). These were in good agreement and the mean was used. For the three very bright calibrating stars, $U B V$ were taken from the Mermilliod, Mermilliod, \& Hauck (1997) on-line database.

We were very fortunate that uniform and reasonably precise broadband infrared photometry at $J H K$, crucial for determination of accurate values of $T_{\text {eff }}$, is now available for all our sample stars from the interim release of the 2MASS

TABLE 2

Observed Colors and Details of the HIRES Spectra for the Stellar Sample

\begin{tabular}{|c|c|c|c|c|c|c|c|c|}
\hline \multicolumn{9}{|l|}{ Very bright: } \\
\hline HD $140283 \ldots \ldots \ldots \ldots \ldots$ & 7.211 & -0.196 & 0.490 & 6.03 & 5.63 & 75 & 300 & 0.000 \\
\hline $\mathrm{BD}+3^{\circ} 740 \ldots \ldots \ldots \ldots$ & 9.808 & -0.20 & 0.36 & 8.77 & 8.49 & 240 & 240 & 0.037 \\
\hline G139-8 .................... & 11.508 & -0.203 & 0.475 & 10.345 & 10.031 & 650 & 165 & 0.067 \\
\hline BS $17447-029 \ldots \ldots \ldots \ldots$ & 13.57 & -0.30 & 0.40 & 12.583 & 12.313 & 1800 & 113 & 0.047 \\
\hline CS 22878-101 ........... & 13.78 & 0.299 & 0.799 & 11.87 & 11.26 & 3600 & 96 & 0.086 \\
\hline CS 22950-046 ........... & 14.224 & 0.345 & 0.906 & 12.228 & 11.570 & 2400 & 93 & 0.108 \\
\hline \multicolumn{9}{|l|}{ HES stars: } \\
\hline HE $2133-1426 \ldots \ldots \ldots$. & 15.484 & -0.237 & 0.449 & 14.374 & 14.120 & 9900 & 105 & 0.052 \\
\hline HE $0148-2611 \ldots \ldots \ldots$ & 14.453 & -0.250 & 0.371 & 13.545 & 13.291 & 3600 & 102 & 0.013 \\
\hline HE $0218-2738^{\mathrm{c}} \ldots \ldots \ldots$ & 14.883 & -0.240 & 0.394 & 13.979 & 13.711 & 7200 & 115 & 0.013 \\
\hline HE $0242-0732 \ldots \ldots \ldots$. & 15.793 & -0.271 & 0.434 & 14.795 & 14.567 & 9300 & 94 & 0.027 \\
\hline
\end{tabular}

a Per spectral resolution element (3 pixels).

b This is a rediscovery of CS 22966-048, originally found in the HK survey.

c The HIRES spectra show that this is a double-lined spectroscopic binary. 


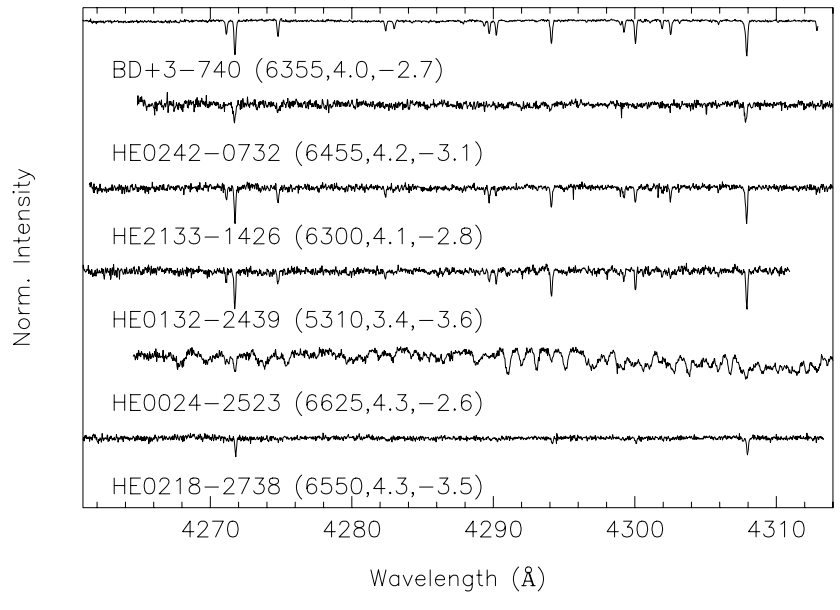

FIG. 1.-HIRES order containing the $\mathrm{G}$ band of $\mathrm{CH}$ for six stars. BD $+3^{\circ} 740$ is a bright calibrating main-sequence star; HE $0242-0732$ is also a turnoff star, as is HE 2133-1426, the faintest star in the present sample. HE $0132-2439$ is the only giant from the HES in our sample. HE $0024-2523$ is the only star that shows easily detectable $\mathrm{CH}$, and its lines are resolved. HE 0218-2738 was found to be a double-lined spectroscopic binary. The spectra have been shifted in wavelength to remove the effects of the difference in radial velocities among these stars.

near-infrared all-sky survey (Skrutskie et al. 1997). The global photometric calibration of this survey is discussed by Nikolaev et al. (2000). We adopt the photometric uncertainties given in the 2MASS catalog. For the bright calibration stars, the near-infrared photometry is from the most recent sources in the compilation of Gezari, Pitts, \& Schmitz (1999), specifically, Arribas \& Martínez-Roger (1987), Laird, Carney, \& Latham (1988), and Alonso, Arribas, \& Martínez-Roger (1994). The photometry is collected in Table 2.

The HES stars are bright enough that preliminary proper motions for most are now available through the US Naval Observatory CCD Astrograph Catalog (Zacharias et al. 2000) for the southern sky, with proper motions for the northern stars in the HES to follow in due course from this same astrometric program. Radial velocities for each EMP candidate from the HES will be obtained as large-scale vetting using moderate-resolution spectroscopy proceeds over the next few years. Thus, the full three-dimensional velocity

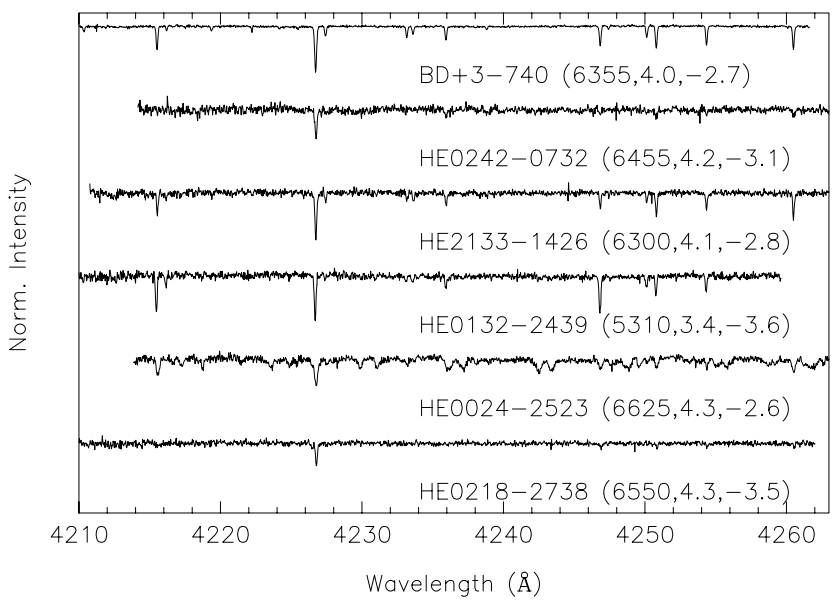

FIg. 2.- Same as Fig. 1, but in the region of the Sr II line at $4215.5 \AA$ A. The strongest line in this order is the $\mathrm{Ca}$ I absorption line at $4226.7 \AA$. The broader lines found in the spectrum of HE 0024-2523 are apparent here. vector can be specified for all the HES metal-poor stars. Such a large nonkinematically selected sample of halo stars will be useful to compare with increasingly sophisticated models of the formation and evolution of the Milky Way, such as those of Chiba \& Beers (2001 and references therein). A first attempt at such a comparison has been presented by Bekki \& Chiba (2001).

Throughout this paper, we use the new $\mathrm{Y}^{2}$ isochrones of Yi et al. (2001), which are calculated for a scaled solar mixture. We adopt an age of $14 \mathrm{Gyr}$ and $[\mathrm{Fe} / \mathrm{H}]=-3.3$ dex, with $Y=0.230$, as the parameters of our standard isochrone. Because the metallicity of our stars is so low, the details of the treatment of the opacities are not critical, and there is reasonably good agreement with isochrones computed using older stellar evolutionary codes such as those of Bergbusch \& VandenBerg (1992). We will find later that the same statement holds true for model atmospheres, for similar reasons.

\section{INTERSTELLAR REDDENING}

A precise measurement of the reddening is crucial for the derivation of accurate stellar parameters from broadband photometry. Even adding the infrared does not help much if the reddening is poorly determined in this $T_{\text {eff }}$ regime. As is shown in Table 4, to be discussed in detail below, the enhanced sensitivity to small changes in $T_{\text {eff }}$ one gains by moving to optical/near-infrared colors such as $V-K$ is accompanied by an enhanced sensitivity to reddening errors. In a project such as mining the HES for EMP stars, where many stars separated by large solid angles on the sky are involved, control of the uniformity of the reddening estimates is mandatory.

We adopt the extinction maps of Schlegel, Finkbeiner, \& Davis (1998) from their analysis of the COBE DIRBE database. The relative extinction in various passbands is taken from Cohen et al. (1981; see also Schlegel et al. 1998).

The HES includes only high Galactic latitude fields within which $E(B-V)$ is almost always $\leq 0.10$ mag. Hence, although there is some concern that the magnitude of the Schlegel et al. extinction corrections may be slightly too large, particularly in regions where the extinction is high $[E(B-V)>0.15$; see, e.g., Arce \& Goodman 1999], we adopt these values without further discussion or amendment.

All the HES stars are fainter than $V \sim 14$, as saturation effects within the photographic plates used for the survey become important for brighter objects. Given their high Galactic latitude, these stars are sufficiently distant, even if they are main-sequence turnoff stars rather than giants, that they can all be assumed to be beyond the reddening layer, whose thickness $H$ is only $\sim 200 \mathrm{pc}$ (see the review by Dickey \& Lockman 1990). Therefore, the full extinction is applied to each of them.

The reddening for the brightest calibrating star (HD 140283 ) is assumed to be 0.00 . This star is only about $60 \mathrm{pc}$ away and has a Hipparcos parallax. The other two calibrating stars and the three stars from the HK survey in the present sample are not so distant as to be beyond the reddening layer, and yet not so close that reddening can be ignored. We therefore use an iterative scheme of estimating the reddening, calculating $T_{\text {eff }}$ (see below), computing the distance $D$ using the luminosity from the stellar evolutionary tracks of Yi et al. (2001), and then checking the estimate 
of the reddening against the quantity $E(B-V) \times$ $\exp [-D \sin (b) / H]$. Similar schemes are described by Laird et al. (1988) and by Bonifacio et al. (2000). Rapid convergence for $E(B-V)$ is obtained.

The recommended values of $E(B-V)$ are given in the last column of Table 2. The reddenings for the HES stars are quite small, with all but one of our sample having $E(B-V)<0.03$. It is only for the brighter calibration objects that this issue becomes a major concern.

\section{6. $T_{\text {eff }}$ FROM BROADBAND COLORS}

Because of concerns about potential non-LTE effects, which are suspected to be stronger in very metal-poor stars than in stars of solar metallicity, we must derive $T_{\text {eff }}$ from broadband colors. Detailed calculations for non-LTE in Fe are presented by Thévenin \& Idiart (1999) and in Gratton et al. (1999). Although the most careful analyses of globular cluster and field stars of higher metallicity than those considered here, such as that of Cohen, Behr, \& Briley (2001) and Ramírez et al. (2001) for a large sample of stars over a wide range in luminosity in M71, Ivans et al. (2001) for a large sample of red giants in M5, or Allende Prieto et al. (2002) for Procyon, show that departures from local thermal equilibrium in the formation of Fe lines are relatively small, the predicted strength of the departures from nonLTE increases as the metallicity decreases. To be conservative, at least initially, measurements based on ionization equilibrium in the spectra themselves cannot be considered reliable at this time.

We utilize here the grid of predicted broadband colors and bolometric corrections from Houdashelt, Bell, \& Sweigart (2000) based on the MARCS stellar atmosphere code (Gustafsson et al. 1975). We assume that their JohnsonGlass $J K$ colors are equivalent to the $J K_{s}$ 2MASS colors. Carpenter (2001) has derived the transformations between the 2MASS photometric system and many other infrared photometric systems and finds a very small zero-point offset between these two infrared photometric systems, which we have chosen to ignore until the final release of the 2MASS catalog becomes available.

Cohen et al. (2001) have demonstrated that the Kurucz and MARCS predicted $V-K$ colors are essentially identical, at least for $V-K$ colors and for the set of models with $[\mathrm{Fe} / \mathrm{H}]=-0.5 \mathrm{dex}$.

Since we are interested in EMP stars, the details of the treatment of the opacity from any element other than hydrogen should not be important, and we expect to continue to find very good agreement for the predicted broadband colors from the various grids of model atmospheres available. In our tests, we take the predicted $V-K$ color from each model in the MARCS grid with $[\mathrm{Fe} / \mathrm{H}]=-3.0$ dex and interpolate within the Kurucz color grid at the same abundance and at the $\log g$ of the MARCS model to find the $T_{\text {eff }}$ that would be deduced. We continue this comparison, checking $V-K, V-J$, and $B-V$ at $[\mathrm{Fe} / \mathrm{H}]=-3.0$ dex. We find that the colors predicted from the MARCS code from Houdashelt et al. are essentially identical to those from the Kurucz ATLAS code (Kurucz 1993) at this low metallicity.

A contour plot of the difference $\Delta T_{\text {eff }}$ (Kurucz minus MARCS) that results when the $V-J$ color is used is shown in Figure 3. The four contour levels displayed correspond to $\Delta T_{\text {eff }}=-30,-10,10$, and $30 \mathrm{~K}$. Also shown in this figure as

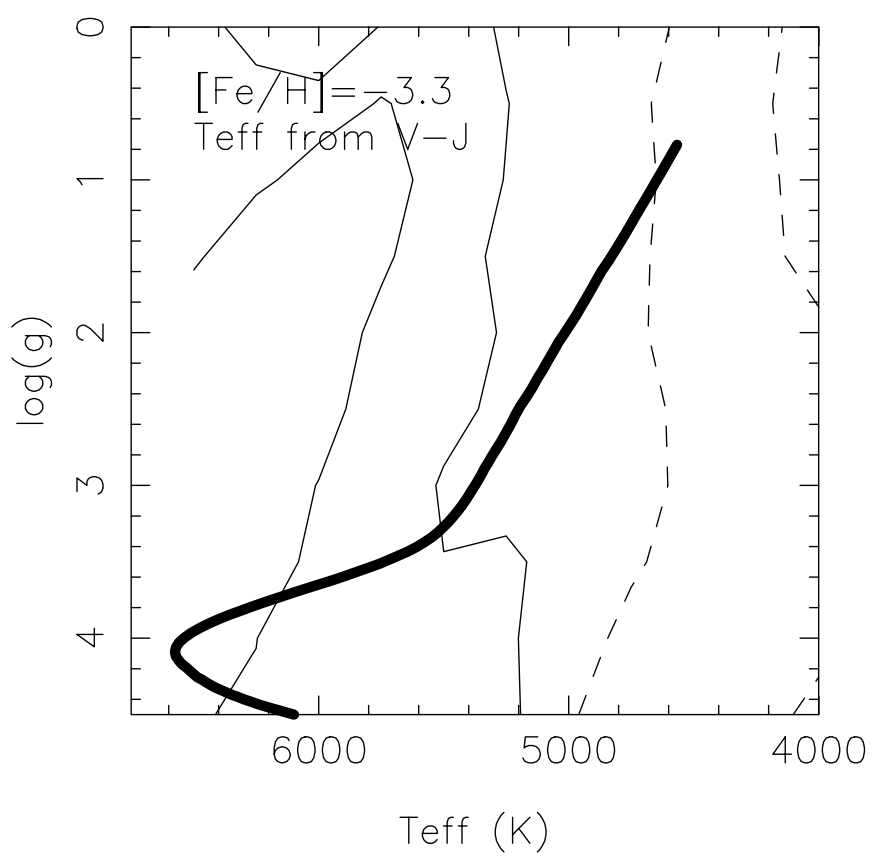

FIG. 3.-Comparison of the Kurucz and MARCS temperature scale from $V-J$ colors. The four contour levels shown correspond to $\Delta T_{\text {eff }}=-30,-10,10$, and $30 \mathrm{~K}$. The thick curve is a $14 \mathrm{Gyr}$ isochrone for a very metal-poor star from the very recently completed $\mathrm{Y}^{2}$ grid of isochrones of Yi et al. (2001).

the thick curve is a $14 \mathrm{Gyr}$ isochrone for $[\mathrm{Fe} / \mathrm{H}]=-3.3$ dex from the very recently completed $\mathrm{Y}^{2}$ isochrones of $\mathrm{Yi}$ et al. (2001). Along this isochrone, $\Delta T_{\text {eff }}=0$ to $30 \mathrm{~K}$ for the subgiants and main-sequence turnoff region, and $\Delta T_{\text {eff }}=0$ to $-30 \mathrm{~K}$ for the red giant branch (RGB). We thus demonstrate that, to within a tolerance of $\pm 30 \mathrm{~K}$, the Kurucz and MARCS temperature scales from broadband $V-J$ colors are identical.

We therefore proceed to assign values of $T_{\text {eff }}$ using the grid of colors from Houdashelt et al. (2000). For the calibrating stars and for some of the brighter stars from the HK survey, a high-dispersion abundance analysis has already been done. These are summarized in Table 3. In these cases, we take the $[\mathrm{Fe} / \mathrm{H}]$ from such an analysis as our initial guess for the stellar metallicity. If not, we assume $[\mathrm{Fe} / \mathrm{H}]=-3.0$ dex. We adopt initial guesses for $\log g$ based on whether the star is a giant or a main-sequence turnoff star. These rough guesses are used to interpolate to the proper value in the desired color, which depends slightly on $\log g$ and abundance, as well as on $T_{\text {eff }}$.

This process is illustrated for a subgiant and for a mainsequence turnoff region star (the faintest star in the present sample) in Figure 4. The results for three colors, $B-V, V-J$, and $V-K$, are shown as solid curves, with the thickness of the curves varying, $B-V$ having the thinnest and $V-K$ having the thickest curves. Dashed curves denote the $T_{\text {eff }}$ deduced for the observed and dereddened colors plus or minus the $1 \sigma$ uncertainty of each observation. In a perfect world, all the solid curves would overlap to within the uncertainties at some definite value of $T_{\text {eff }}$, and this does in fact occur for the stars shown in the figure. We have found that the $B-V$ colors give systematically higher $T_{\text {eff }}$, by about $50 \mathrm{~K}$, for the main-sequence EMP stars (see Fig. 4).

Table 4 gives the sensitivity of each color to various stellar parameters. These are expressed as the change in the param- 
TABLE 3

Recent High-Dispersion Abundance Analyses of THE BRIGHTER SAMPLE STARS

\begin{tabular}{|c|c|c|c|c|c|}
\hline Name & $\begin{array}{l}T_{\text {eff }} \\
(\mathrm{K})\end{array}$ & $\begin{array}{l}\log g \\
(\operatorname{dex})\end{array}$ & $\begin{array}{c}{[\mathrm{Fe} / \mathrm{H}]} \\
(\mathrm{dex})\end{array}$ & $\begin{array}{c}E(B-V) \\
(\mathrm{mag})\end{array}$ & Ref. \\
\hline \multicolumn{6}{|l|}{ Very bright: } \\
\hline \multirow[t]{4}{*}{ HD $140283 \ldots \ldots \ldots . .}$. & 5750 & 3.4 & -2.54 & 0.01 & 1 \\
\hline & 5680 & 3.5 & -2.64 & 0.00 & 2 \\
\hline & 5810 & 3.67 & -2.29 & $\mathrm{a}$ & 3 \\
\hline & 5650 & 3.4 & -2.4 & b & 4 \\
\hline \multirow[t]{2}{*}{$\mathrm{BD}+3^{\circ} 740 \ldots \ldots \ldots$} & 6240 & $4^{c}$ & -2.70 & 0.01 & 5 \\
\hline & 6075 & 3.8 & -2.7 & $\mathrm{~b}$ & 4 \\
\hline G139-8 ……............. & 6025 & $4^{c}$ & -2.56 & 0.04 & 6 \\
\hline \multicolumn{6}{|l|}{ HK stars: } \\
\hline CS 22878-101 ........ & 4780 & 1.15 & -3.13 & 0.06 & 7 \\
\hline CS 22950-046 ........ & 4635 & 0.85 & -3.40 & 0.06 & 7 \\
\hline
\end{tabular}

${ }^{\text {a }} T_{\text {eff }}$ based on analysis of Balmer line profiles.

b $T_{\text {eff }}$ deduced from Fe spectrum.

c Assumed to be at or near the main-sequence turnoff.

REFERENCES.- (1) Ryan et al. 1996; (2) Nissen et al. 1994 and Edvardsson et al. 1994; (3) Korn \& Gehren 2001, using $\log g$ from Hipparcos parallax; (4) Fulbright 2000; (5) Ryan, et al. 1999; (6) Norris et al. 1997; (7) McWilliam et al. 1995.

eter (which may be positive or negative) that would result from $\mathrm{a}+1 \sigma$ change in the color, where the observational uncertainty for each color defines the value of $\sigma$ used. These calculations were made using stellar parameters appropriate for EMP main-sequence turnoff stars. We infer from this table, for example, that $B-V$ is less sensitive to changes in

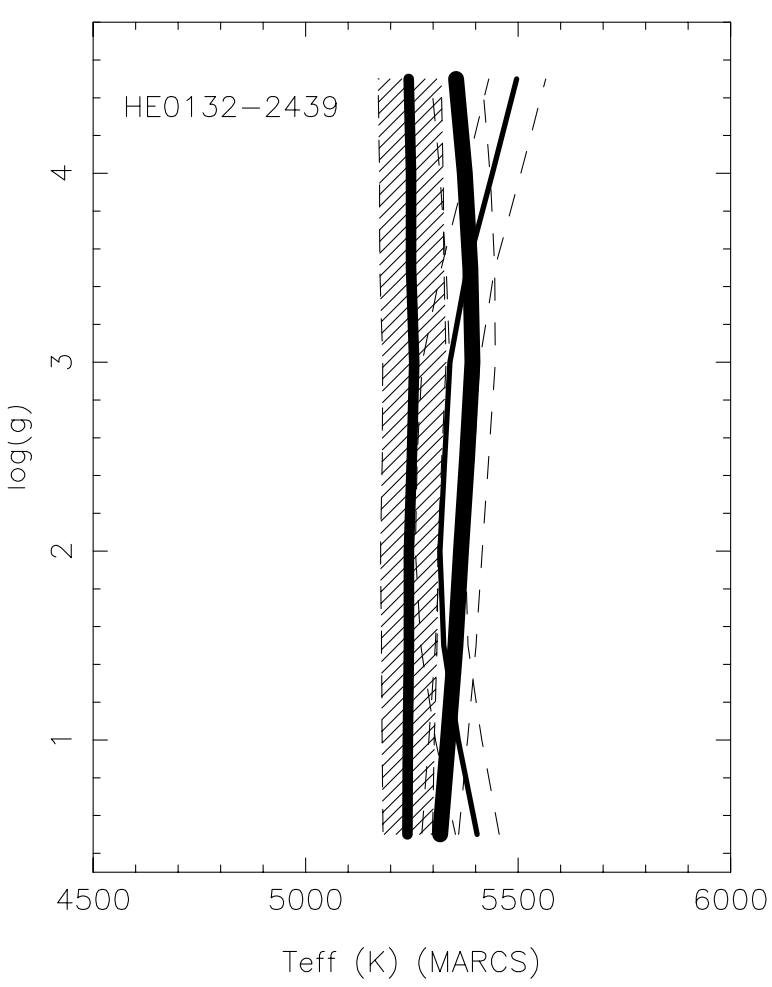

$T_{\text {eff }}$ than either $V-J$ or $V-K$, and that none of the colors considered are capable of applying a significant constraint on the metallicity of a star.

The values of $T_{\text {eff }}$ that result from this procedure, applied to each star in our sample, are listed in Table 5. As one might expect from Table 4, they have typical internal errors of $\pm 75 \mathrm{~K}$, except for those from $B-V$, where the error is about $\pm 125 \mathrm{~K}$.

$U-B$ colors are also available, but they are more difficult to match to the predictions of the model atmosphere grid. For five of the eight stars from the HES, $U-B<-0.25$ when corrected for reddening. This is very blue, so much so that such a blue color is not reached in the relevant regime of $T_{\text {eff }}$ and $\log g$, even at $[\mathrm{Fe} / \mathrm{H}]=-3.0$ dex, the most metal-poor models in the grid of Houdashelt et al. (2000). In addition, the predictions for $U-B$ depend sensitively on metallicity as well as on $T_{\text {eff }}$ and $\log g$, and they are nonmonotonic in these two parameters.

While the contribution from line opacities becomes less important in defining continuum fluxes at the lowest metallicities, some problems in the violet and ultraviolet may remain. We believe that these problems of properly predicting the flux in the region $3500-4500 \AA$ in these EMP stars are related to the extremely low metallicity of these stars, to the sensitivity to multiple parameters, and to the relatively small sensitivity to $T_{\text {eff. }}$. This situation is alleviated by using redder colors with wider wavelength separation. Therefore, the adopted $T_{\text {eff }}$ values given in Table 5 are the means of those deduced from the dereddened $V-J$ and the $V-K$ colors.

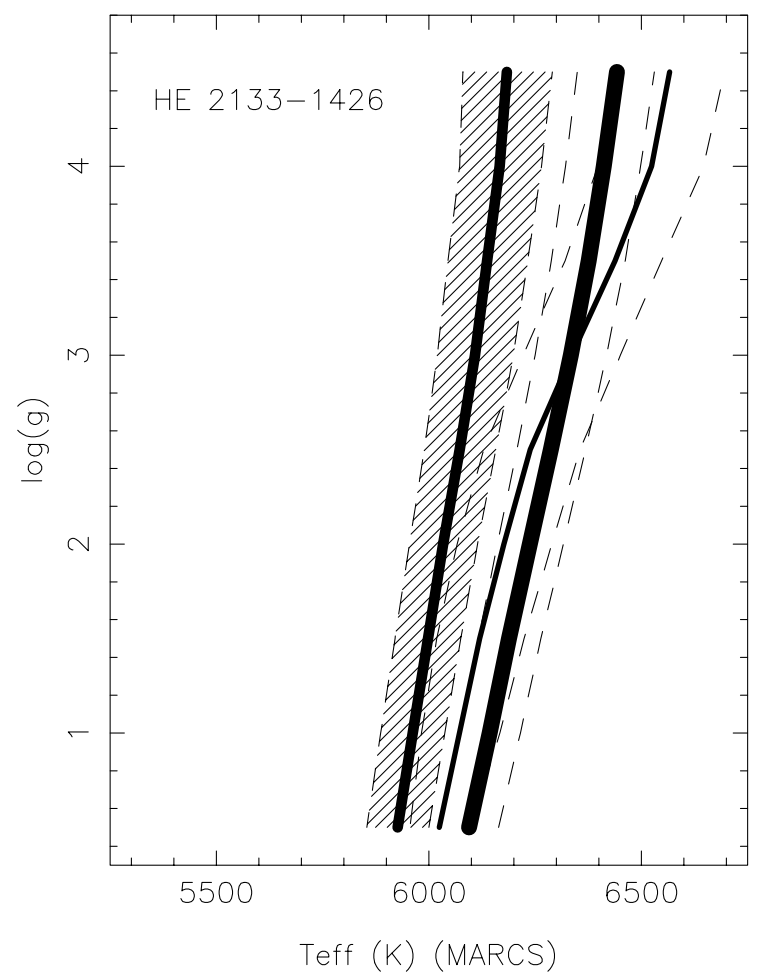

FIG. 4.-Examples of our procedure for deriving $T_{\text {eff }}$ from stars in our sample. Left: HE 0132-2439, an EMP giant. The solution for its $T_{\text {eff }}$ from its dereddened colors is displayed as a function of $\log g$, with $[\mathrm{Fe} / \mathrm{H}]$ assumed to be -3.0 dex. The results from the $B-V$ measurement are shown as the thinnest solid curve, from the $V-J$ color as thicker solid curve, and from $V-K$ as the thickest solid curve. Dashed curves denote the values of $T_{\text {eff }}$ inferred from the measured and dereddened colors plus or minus their $1 \sigma$ uncertainties. The hatched area indicates that allowed within this $1 \sigma$ uncertainty level inferred from the $V-J$ color. Right: The $T_{\text {eff }}$ determination for the faintest star in the present sample, HE 2133-1426, an EMP star near the main-sequence turnoff. 
TABLE 4

SENSITIVITY OF THE VARIOUS COLORS FOR EMP TURNOFF STARS

\begin{tabular}{cccccc}
\hline \hline Color & $\begin{array}{c}1 \sigma^{\mathrm{a}} \\
(\mathrm{mag})\end{array}$ & $\begin{array}{c}\delta T_{\text {eff }}^{\mathrm{b}} \\
(\mathrm{K})\end{array}$ & $\begin{array}{c}\delta \log g^{\mathrm{b}} \\
(\mathrm{dex})\end{array}$ & $\begin{array}{c}\delta[\mathrm{Fe} / \mathrm{H}]^{\mathrm{b}} \\
(\mathrm{dex})\end{array}$ & $\begin{array}{c}\delta E(B-V)^{\mathrm{b}} \\
(\mathrm{mag})\end{array}$ \\
\hline$B-V \ldots \ldots \ldots$ & 0.02 & -125 & +1.4 & $>2.0$ & +0.020 \\
$V-J \ldots \ldots \ldots$. & 0.03 & -71 & +1.7 & $>2.0$ & +0.014 \\
$V-K \ldots \ldots \ldots$ & 0.04 & -73 & +1.7 & $>2.0$ & +0.015 \\
\hline
\end{tabular}

a Typical $1 \sigma$ observational errors.

${ }^{\mathrm{b}}$ Change in the stellar parameter for $\mathrm{a}+1 \sigma$ change in the color.

We have also determined $T_{\text {eff }}$ through examination of the Balmer line profiles. $\mathrm{H} \alpha$ is the Balmer line best suited for this purpose, because it has the strongest temperature sensitivity and its profile is almost independent of the choice of the mixing-length parameter $\alpha$ (see, e.g., Fuhrmann, Axer, \& Gehren 1993). Unfortunately, $\mathrm{H} \alpha$ is not covered by the spectral range chosen in this work. $\mathrm{H} \beta$ is not well centered in the echelle orders and is hence unsuitable. The continuum near $\mathrm{H} \gamma$ may be perturbed by strong $\mathrm{CH}$ lines in carbonenhanced stars, which occur at a rate of $\sim 20 \%$ among EMP stars. Hence we utilize $\mathrm{H} \delta$ for this purpose. A special reduction of the relevant order was made to ensure the best possible continuum level. The orders above and below this were used as necessary to interpolate the continuum level across the Balmer line. The synthetic Balmer line profiles computed by T. Gehren's group (Fuhrmann 1998; K. Fuhrmann 2000, private communication) are used. The results are listed in the fifth column of Table 5. These have a typical error of $\pm 100 \mathrm{~K}$.

A perusal of Table 5 shows that we have achieved consistency to within $\pm 100 \mathrm{~K}$ between the $T_{\text {eff }}$ from the Balmer line profile and from the broadband colors, as is also shown in Figure 5. The solid line denotes equality between the $T_{\text {eff }}$ from the broadband photometry and from the $\mathrm{H} \delta$ profiles, while the dashed line is the best linear fit excluding the single subgiant, which suggests that the $T_{\text {eff }}$ for the turnoff stars from the broadband photometry is systematically slightly hotter than that inferred from $\mathrm{H} \delta$.

We note that the $T_{\text {eff }}$ we assign to these main-sequence turnoff stars is identical to that found through stellar evolution, that is, the predicted location of the main-sequence turnoff in 14 Gyr isochrones for metal-poor stars, which is at about $6600 \mathrm{~K}$ for $[\mathrm{Fe} / \mathrm{H}]=-3.3 \mathrm{dex}$ and about $6500 \mathrm{~K}$ for $[\mathrm{Fe} / \mathrm{H}]=-2.3$ dex. If the $T_{\text {eff }}$ we assign are consistent with the $T_{\text {eff }}$ scale adopted for the $\mathrm{Y}^{2}$ grid of isochrones, ages older than 14 Gyr can be ruled out, as the main-sequence turnoff then becomes cooler than the observed values reached by these stars. Such old ages are also not supported by recent BOOMERANG observations of the fluctuations in the cosmic microwave background (de Bernardis et al. 2000).

\subsection{Comparison with the Empirical $T_{\mathrm{eff}}$ Scale of Alonso et al.}

We have compared our $T_{\text {eff }}$ scale with the empirical color$T_{\text {eff- }}[\mathrm{Fe} / \mathrm{H}]$ relations for dwarfs and for giants established by Alonso, Arribas, \& Martínez-Roger (1996, 1999). We use their polynomial fit for the $V-K$ colors, simulating moving down in luminosity along an isochrone from the tip of the RGB to the main sequence for EMP stars. Since the Alonso et al. fits are not well calibrated at extremely low metallicities, we use their fits with $[\mathrm{Fe} / \mathrm{H}]=-2.0$ dex; the metallicity sensitivity of $V-K$ at lower abundances than this is small.

TABLE 5

Stellar Parameters for the Stellar Sample

\begin{tabular}{|c|c|c|c|c|c|c|}
\hline \multirow[b]{2}{*}{ NAME } & \multicolumn{5}{|c|}{$T_{\text {eff }}(\mathrm{K})$} & \multirow{2}{*}{$\begin{array}{l}\log g \\
(\mathrm{dex})\end{array}$} \\
\hline & $B-V$ & $V-J$ & $V-K$ & $\mathrm{H} \delta$ & Adopted $^{\mathrm{a}}$ & \\
\hline \multicolumn{7}{|l|}{ Very bright: } \\
\hline HD $140283 \ldots \ldots \ldots \ldots \ldots . . . . .$. & 5935 & 5710 & 5785 & 5800 & 5750 & 3.67 \\
\hline $\mathrm{BD}+3^{\circ} 740 \ldots \ldots \ldots \ldots$ & 6750 & 6260 & 6450 & 6400 & 6355 & 4.0 \\
\hline G139-8 ...................... & 6500 & 6105 & 6290 & 6200 & 6200 & $4.5^{\mathrm{b}}$ \\
\hline \multicolumn{7}{|l|}{ HK stars: } \\
\hline BS 17447-029............ & 6800 & 6470 & 6570 & $\ldots$ & 6530 & $4.4^{\mathrm{b}}$ \\
\hline CS 22878-101 ............. & 4870 & 4735 & 4820 & $\mathrm{c}$ & 4775 & 1.3 \\
\hline CS 22950-046 ............ & 4870 & 4695 & 4770 & $\mathrm{c}$ & 4730 & 1.3 \\
\hline \multicolumn{7}{|l|}{ HES stars: } \\
\hline HE 2133-1426 ......... & 6475 & 6145 & 6460 & 6300 & 6300 & 4.1 \\
\hline HE $2344-2800^{\mathrm{d}} \ldots \ldots .$. & 6700 & 6500 & 6750 & 6400 & 6625 & 4.3 \\
\hline HE $0024-2523 \ldots \ldots \ldots$. & 6550 & 6700 & 6550 & 6500 & 6625 & 4.3 \\
\hline HE $0130-2303 \ldots \ldots \ldots$. & 6600 & 6600 & 6525 & 6500 & 6560 & 4.3 \\
\hline HE $0132-2439 \ldots \ldots \ldots$. & 5440 & 5250 & 5375 & 5400 & 5310 & 3.4 \\
\hline HE $0148-2611 \ldots \ldots \ldots$. & 6800 & 6500 & 6600 & 6500 & 6550 & 4.3 \\
\hline HE $0218-2738^{\mathrm{e}} \ldots \ldots \ldots$ & 6625 & 6500 & 6600 & 6400 & 6550 & 4.3 \\
\hline HE $0242-0732 \ldots \ldots \ldots$. & 6550 & 6360 & $6550^{f}$ & 6200 & 6455 & 4.2 \\
\hline
\end{tabular}

a Adopted $T_{\text {eff }}$ is a mean of that derived from the dereddened $V-J$ and $V-K$ color; see text.

b The lower luminosity solution is chosen (see Paper II), so the star is fainter than the main-sequence turnoff; see text.

c Star is too cool to show Balmer line wings.

$\mathrm{d}$ This is a rediscovery of CS 22966-048, originally found in the HK survey.

e The HIRES spectra show that this is a spectroscopic binary.

f The uncertainty in the 2 MASS $K$ magnitude is unusually large, $1 \sigma=0.10 \mathrm{mag}$. 


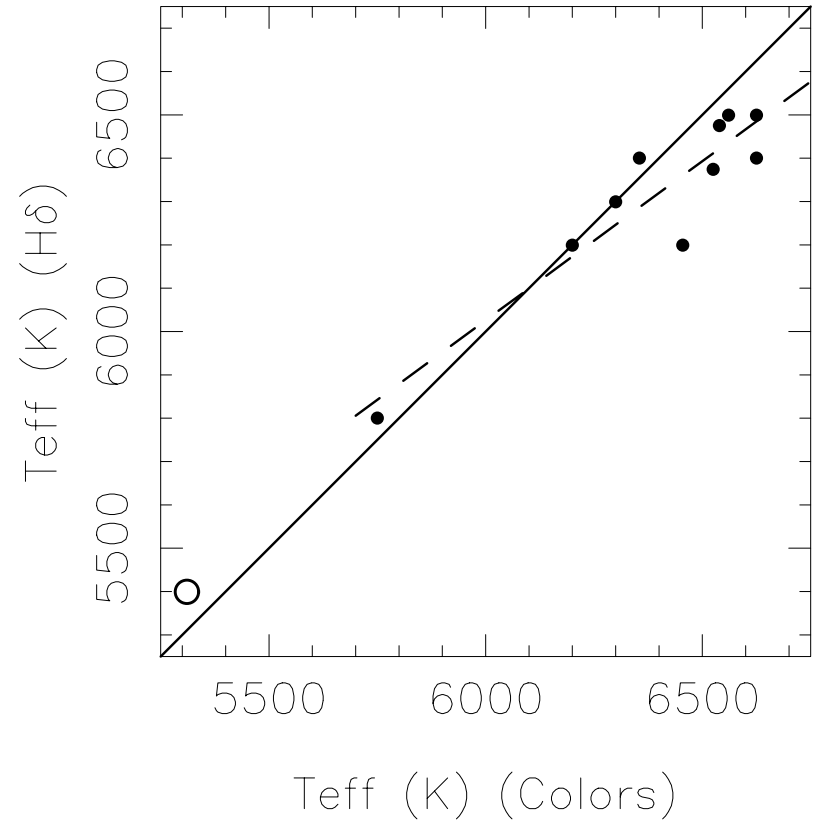

FIG. 5.- $T_{\text {eff }}$ values deduced from the analysis of the profiles of the $\mathrm{H} \delta$ lines in our sample of very metal-poor stars as a function of the $T_{\text {eff }}$ values derived from the observed, dereddened colors. The solid line denotes equality, while the dashed line indicates the best linear fit. The large open circle denotes the only subgiant in our present sample, which is not included in the fit. The $\sigma$ about the best-fit line is $\sim 75 \mathrm{~K}$.

We find that $T_{\text {eff }}$ inferred from the Alonso et al. relations for the $V-K$ color is $150 \mathrm{~K}$ cooler than that inferred from the Houdashelt et al. (2000) grid of colors predicted from stellar atmosphere models near the top of the giant branch, but this decreases to $100 \mathrm{~K}$ cooler for subgiants and for stars near the main-sequence turnoff for $[\mathrm{Fe} / \mathrm{H}]=-2.0$ dex. Pushing the Alonso et al. relations beyond their regime of validity and verification to $[\mathrm{Fe} / \mathrm{H}]=-3.0$ dex would produce good agreement at the main sequence, but the subgiants and giants would still be $100 \mathrm{~K}$ hotter using the MARCS color grid. ${ }^{7}$ The Alonso scale thus is close to the $T_{\text {eff }}$ determined from $\mathrm{H} \delta$.

Thus, at least with respect to the $V-K$ color, the Alonso et al. scale and our $T_{\text {eff }}$ scale are in good agreement for turnoff stars. The small systematic difference of a maximum of $100 \mathrm{~K}$ for giants would translate into a change in derived metallicity of $\Delta[\mathrm{Fe} / \mathrm{H}] \sim-0.1$ dex.

Because the Alonso et al. empirical $T_{\text {eff }}$ scale is not calibrated for stars as metal-poor as our sample, and because of our philosophical preference to utilize the same model atmospheres that one must rely upon for analyses of the spectral features, we have chosen to adopt the $T_{\text {eff }}$ scale established from broadband optical-infrared colors. One potential systematic error for this choice is the matching of the photometric systems between the observational colors and the theoretically predicted ones. We believe that the internal errors in our adopted $T_{\text {eff }}$ are $\pm 75 \mathrm{~K}$, with a possible systematic overestimate (arising from as yet unknown causes) for the main-sequence turnoff stars only of $\sim 100 \mathrm{~K}$.

\footnotetext{
${ }^{7}$ This may be related to problems encountered by Lebreton (2000) in matching stellar evolutionary isochrones with stellar parameters for nearby stars with Hipparcos parallaxes.
}

\subsection{Comparison with Previous $T_{\mathrm{eff}}$ Determinations}

We compare our values of $T_{\text {eff }}$ with the limited set of values available from previous analyses of the brighter stars in our sample. We adjust the $T_{\text {eff }}$ values listed in Table 5 for the difference in reddening between the values we adopt given in Table 2 and those adopted in previous analyses. The results are given in Table 6 . Once the reddenings are forced to the same value, each of the five entries has $\mid \Delta T_{\text {eff }}($ old - new $) \mid<$ $100 \mathrm{~K}$. This is very encouraging.

\section{SURFACE GRAVITIES}

The only broadband color among those available to us with strong sensitivity to $\log g$ is $U-B$. However, for the reasons described in $\S 6$, we choose not to use it except in the crudest possible sense, that is, to discriminate among turnoff stars and subgiants and giants. We cannot utilize the usual spectroscopic indicator, the ionization equilibrium, because of the possibility of non-LTE effects, as described above.

Once $T_{\text {eff }}$ is known, we determine $\log g$ from the $\mathrm{Y}^{2}$ isochrone for stars that are $14 \mathrm{Gyr}$ old with $Z=1.0 \times 10^{-5}$, equivalent to $[\mathrm{Fe} / \mathrm{H}]=-3.3$ dex. The $\log g$ is determined directly from the isochrone using the $T_{\text {eff }}$ of the star. If the $12 \mathrm{Gyr}$ isochrone of the same metallicity from the $\mathrm{Y}^{2}$ grid were adopted, the assigned $\log g$ would decrease by an amount that is negligible for the main-sequence stars and which decreases as $T_{\text {eff }}$ decreases, with a difference of $\sim 0.15$ dex at $T_{\text {eff }}=5000 \mathrm{~K}$.

This procedure is not very precise for giants, because the slope of the RGB is rather steep, $\Delta\left[\log (g) / T_{\text {eff }}\right] \sim 0.3$ dex per $100 \mathrm{~K}$. Consideration of the rate of evolution along the isochrone as a function of luminosity, which directly translates into the number density of stars along the isochrone, dictates that the probability of finding subgiants is considerably higher in faint samples such as the HES than that of finding stars well up the RGB.

For stars near the main-sequence turnoff, which is the location of the bulk of the present sample, the slope of the isochrone is much shallower and good discrimination in $\log g$ is possible. However, there are two solutions in the isochrone for a fixed $T_{\text {eff }}$ near the main-sequence turnoff, one corresponding to main-sequence stars slightly fainter than the turnoff, and one to stars that have just started to evolve off the main sequence toward the base of the giant branch; these two solutions differ by about 1 dex in $\log g$ for $T_{\text {eff }} \sim 5900 \mathrm{~K}$. For $T_{\text {eff }}$ more than $\sim 500 \mathrm{~K}$ cooler than the turnoff itself, the lower luminosity of the main-sequence stars makes their detection quite unlikely, even though their volume density is higher than the post-turnoff mainsequence stars. In fact, assuming a constant volume density over the Galactic region sampled by the HES, ${ }^{8}$ a comparison with globular cluster luminosity functions shows that at this temperature, we expect to detect approximately one main-sequence star out of 10-15 subgiants. For $T_{\text {eff }}$ closer to the turnoff, both solutions have fairly similar probabilities, although the brighter (lower gravity) solution is always more probable.

We cannot distinguish between these two cases using our present photometric data set alone. Making this distinction

\footnotetext{
${ }^{8}$ Typical distances of turnoff stars in the HES are $\sim 1 \mathrm{kpc}$; this is much less than the local value of the halo scale height.
} 
TABLE 6

Comparison of $T_{\text {eff }}$ Determinations

\begin{tabular}{|c|c|c|c|c|c|}
\hline Name & $\begin{array}{l}T_{\text {eff }} \\
(\mathrm{K})\end{array}$ & $\begin{array}{l}E(B-V) \\
(\mathrm{mag})\end{array}$ & Refs. & $\begin{array}{c}T_{\text {eff }}(\text { Table } 3) \\
(\mathrm{K})\end{array}$ & $\begin{array}{c}T_{\text {eff }}[\text { Table } 3 \text { with Previous } \\
E(B-V)]^{\mathrm{a}}\end{array}$ \\
\hline \multicolumn{6}{|l|}{ Very bright: } \\
\hline HD $140283 \ldots \ldots \ldots \ldots$ & $5680-5810$ & 0.01 & $1,2,3,4$ & 5750 & 5785 \\
\hline $\mathrm{BD}+3^{\circ} 740 \ldots \ldots \ldots \ldots$ & 6240 & 0.01 & 5 & 6355 & 6260 \\
\hline G139-8 …............... & 6025 & 0.04 & 6 & 6200 & 6105 \\
\hline \multicolumn{6}{|l|}{ HK stars: } \\
\hline CS 22878-0101_........ & 4780 & 0.06 & 7 & 4775 & 4730 \\
\hline CS 22950-0046........ & 4635 & 0.06 & 7 & 4730 & 4620 \\
\hline
\end{tabular}

${ }^{\text {a }}$ Here we derive $T_{\text {eff }}$ using our photometry and calibrations but adopt the reddening value used in the previous abundance determination.

REFERENCES. - (1) Ryan et al. 1996; (2) Nissen et al. 1994 and Edvardsson et al. 1994; (3) Korn \& Gehren 2001, using $\log g$ from Hipparcos parallax; (4) Fulbright 2000; (5) Ryan et al. 1999; (6) Norris et al. 1997; (7) McWilliam et al. 1995.

using the Fe ionization equilibrium (i.e., relying on the spectra, and on the understanding of or absence of large nonLTE effects) is only feasible in the best of our spectra, as the difference in $\log g$ between the two solutions is not large. As noted in Table 5, the detailed analysis of the spectra presented in Paper II suggests that two of the three bright comparison stars near the main-sequence turnoff actually are slightly fainter than the turnoff. All the HES sample members have been assigned to be brighter than the mainsequence turnoff.

Only one of the stars among the bright calibration objects has an accurate Hipparcos parallax, HD 140283, discussed by Korn \& Gehren (2001). We adopt their value of $\log g$. Our derived $\log g$ for $\mathrm{BD}+3^{\circ} 740$ is consistent with that inferred from the rather uncertain Hipparcos parallax for that star (7.8 \pm 2.1 mas), assuming a mass of $0.8 M_{\odot}$.

We emphasize that our $T_{\text {eff }}$ determination is independent of any spectroscopic criteria, and that the choice of $\log g$ is made based on $T_{\text {eff }}$ and an assumed isochrone. It is only when there are multiple possible values of $\log g$ with approximately equal probability, which occurs only near the main-sequence turnoff, that spectroscopic criteria are used. The difference in $\log g$ between the two solutions does not exceed 0.5 dex before the lower luminosity solution becomes highly improbable. The details of the abundance analyses presented in Paper II verify the validity of this process.

\section{CAUTIONARY NOTES}

Kurucz (2002) has reviewed the many deficiencies of the current state of stellar astrophysics. The treatment of nonLTE in essentially all abundance analyses may be inadequate. (We only apply non-LTE corrections for a selected small set of ions; see Paper II.) Our treatment of convection surely could be better. Detailed three-dimensional radiative-hydrodynamic convection simulations for solar granulation such as those of Asplund et al. (2000) now exist. The first steps toward utilizing such models (Allende-Prieto et al. 2001) to study line profiles and abundances are now starting to become available, although such an elegant treatment still requires large amounts of computing time and very high precision spectra, with much better spectral resolution and $\mathrm{S} / \mathrm{N}$ than those discussed here.

In our present application, the question is how well the continuum and its broadband colors, or the Balmer line profiles, represent the temperature gradient within the atmosphere. This is what is explored by the absorption features, as strong lines tend to arise from levels with smaller excitation potentials and hence are formed farther out in the atmosphere. Kurucz points out that Balmer line wings will be preferentially formed in the hotter convective elements as a consequence of the high excitation potential of the second level of $\mathrm{H}$ I. Hence a simple one-dimensional model such as we are using will have a somewhat higher $T_{\text {eff }}$ deduced from the Balmer lines than does the real star. However, with such metal-poor stars, all the absorption lines are very weak, and the issue of the temperature gradient within the atmosphere is perhaps less critical, as the lines are formed closer to the mean depth of formation of the continuum.

$T_{\text {eff }}$ obtained from the excitation temperature of $\mathrm{Fe}$ should be reliable, and disagreements between these atmospheric parameters and those from $T_{\text {exc }}$ might indicate problems with the correct temperature stratification in the atmosphere. Such problems have been noted in the past by Dalle Ore (1992) and Gratton, Carretta, \& Castelli (1996), with a more limited discussion given by Fulbright (2000). These problems appear to be considerably worse in the very metal-poor giants than in the dwarfs. This issue will be taken up again in Paper II.

We have adopted a prescription, based on photometric indices for determining the stellar parameters of our very metal-poor stars, that is quite different from that used in most previous analyses, which rely much more on spectroscopic equilibria and/or exclusively on bluer colors. By rejecting the spectroscopic equilibria in choosing $T_{\text {eff }}$, and by using theoretical isochrones to determine $\log g$, we leave open the possibility that our derived $\mathrm{Fe}$ abundances may have a dependence on the lower excitation potential of the transition, $\chi$. This in fact turns out to be the case; we do find a small dependence in some stars in Paper II. The possible existence of this dependence means that care must be exercised in comparing our abundances with those from other analyses, as the distribution of line excitation potentials in the two samples must be taken into account.

\section{THE YIELD OF THE HES FOR EMP STARS}

In the last column of Table 7, we give the Fe abundance $([\mathrm{Fe} / \mathrm{H}]$ deduced from $\mathrm{Fe}$ I lines) for the present sample, taken from Paper II. For the bright stars with previous high-dispersion abundance analyses, listed in Table 3, we 
TABLE 7

Spectroscopic Parameters for the Stellar Sample

\begin{tabular}{|c|c|c|c|}
\hline Name & $\begin{array}{c}v_{r}^{\mathrm{a}} \\
\left(\mathrm{km} \mathrm{s}^{-1}\right)\end{array}$ & $\begin{array}{c}{[\mathrm{Fe} / \mathrm{H}]_{\mathrm{K}}^{\mathrm{b}}} \\
(\mathrm{dex})\end{array}$ & $\begin{array}{c}{[\mathrm{Fe} / \mathrm{H}]_{\text {HIRES }}{ }^{\mathrm{c}}} \\
(\mathrm{dex})\end{array}$ \\
\hline \multicolumn{4}{|l|}{ Very bright: } \\
\hline HD $140283 \ldots \ldots \ldots \ldots \ldots$ & $-171.0^{\mathrm{d}}$ & $\ldots$ & -2.43 \\
\hline $\mathrm{BD}+3^{\circ} 740 \ldots \ldots \ldots \ldots$ & +174.5 & $\ldots$ & -2.69 \\
\hline G139-8 …................. & -114.0 & $\ldots$ & -2.04 \\
\hline \multicolumn{4}{|l|}{ HK stars: } \\
\hline BS 17447-029............. & -205.0 & $-3.15(0.26)$ & -2.91 \\
\hline 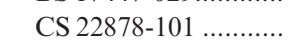 & -131.7 & $-2.91(0.18)$ & -3.07 \\
\hline CS 22950-046 ............ & +106.7 & $-3.63(0.15)$ & -3.27 \\
\hline \multicolumn{4}{|l|}{ HES stars: } \\
\hline HE $2133-1426 \ldots \ldots \ldots$ & +19.6 & $-3.32(0.23)$ & -2.81 \\
\hline HE $2344-2800 \ldots \ldots \ldots$. & -135.8 & $-3.06(0.20)$ & -2.53 \\
\hline HE $0024-2523^{\mathrm{e}} \ldots \ldots \ldots$ & -181.6 & $-3.08(0.24)$ & -2.62 \\
\hline HE $0130-2303 \ldots \ldots \ldots$. & +74.2 & $-3.15(0.19)$ & -2.93 \\
\hline HE $0132-2439 \ldots \ldots \ldots$. & +294.7 & $-3.05(0.33)$ & -3.56 \\
\hline HE $0148-2611 \ldots \ldots . .$. & -223.1 & $-3.42(0.17)$ & -2.96 \\
\hline HE $0218-2738^{\mathrm{f}} \ldots \ldots \ldots$ & +134.0 & $-3.81(0.17)$ & -3.52 \\
\hline HE $0242-0732 \ldots \ldots \ldots$. & -188.9 & $-3.59(0.20)$ & -3.04 \\
\hline
\end{tabular}

a Heliocentric radial velocity.

$\mathrm{b}$ These values are inferred from the strength of the $\mathrm{Ca}$ II $\mathrm{K}$ line and the Balmer lines in the moderate-resolution spectra. There is a small systematic offset of 0.23 dex between the abundance scales of the follow-up spectra and of the high-resolution spectra. See text for details.

${ }^{\mathrm{c}}[\mathrm{Fe} / \mathrm{H}]$ values are from the detailed abundance analysis presented in Paper II.

d The uncertainty in the radial velocities is dominated by systematic errors and is $\pm 1.0 \mathrm{~km} \mathrm{~s}^{-1}$.

${ }^{\mathrm{e}}$ Has easily detectable $\mathrm{CH}$ absorption in the $\mathrm{G}$ band, and its lines are resolved.

${ }^{\mathrm{f}}$ The HIRES spectra show that this is a double-lined spectroscopic binary.

find that our abundances are comparable when the $T_{\text {eff }}$ values adopted in both analyses are essentially identical (see Table 6) and tend to be slightly higher in those cases where our adopted $T_{\text {eff }}$ is hotter, as expected.

Three of the eight HES candidate EMP stars in the present sample do in fact have $[\mathrm{Fe} / \mathrm{H}] \leq-3.0 \mathrm{dex}$, with three others having $[\mathrm{Fe} / \mathrm{H}] \leq-2.8$ dex. The sample of two very metal-poor stars observed by Depagne et al. (2000) at the VLT also yielded metallicities close to those expected from their moderate-resolution follow-up spectra. This means that the process of automatic selection of EMP candidates from the HES, followed by a visual check and then further vetting using moderate-resolution spectroscopy, produces samples of EMP stars with a high yield.

It is interesting to compare our derived $[\mathrm{Fe} / \mathrm{H}]$ values from the present set of HIRES spectra with the abundance inferred for the stars in our sample from the moderateresolution follow-up spectroscopy. Those values are deduced from the strengths of the Ca II absorption at 3933 $\AA$, the Balmer lines, and the $B-V$ colors with the standard method developed for the HK survey, and most recently described in Beers et al. (1999), ${ }^{9}$ and are given in the nextto-last column of Table 7, together with a $1 \sigma$ error on the abundance determination. The metallicities obtained from

\footnotetext{
${ }^{9}$ Some additional features, such as autocorrelation function measurements, were added to the standard abundance estimation algorithms for the HK survey by Beers et al. (1999); those were not used for the HES stars.
}

the moderate-resolution spectra are, in general, somewhat more metal-poor, part of which may arise from the somewhat hotter $T_{\text {eff }}$ values adopted here. The difference in the mean $[\mathrm{Fe} / \mathrm{H}]_{\mathrm{K}}-[\mathrm{Fe} / \mathrm{H}]_{\text {HIRES }}$ is -0.27 dex, with a dispersion about this mean offset of $0.33 \mathrm{dex}$. This dispersion is in accordance with expectation, given the listed errors in $[\mathrm{Fe} / \mathrm{H}]_{\mathrm{K}}$ and the smaller errors expected for the present effort. A detailed discussion of these moderate-resolution spectra and the derivation of metallicities therefrom will be given elsewhere (Christlieb et al. 2002).

All this is very encouraging for future large-scale projects that plan to mine the HES database for extremely metalpoor stars.

\section{SUMMARY}

In this paper and its companion, which presents a detailed abundance analysis for these HIRES spectra, we have demonstrated the high effective yield of the HES for extremely metal-poor stars, and we now intend to embark on a major project (the $0 \mathrm{Z}$ Project) to understand the early chemical evolution of the Galactic halo.

Our ultimate goal, which will take perhaps 5 years to reach with several large observatories working in a coordinated fashion, is high-dispersion abundance analyses of a sample of 500 extremely metal-poor stars. We will gain a detailed knowledge of chemical evolution during the initial phases of the formation of the Galactic halo, the kinematic properties of the extremely metal-poor halo stars, a much better knowledge of the metallicity distribution of very metal-poor halo stars, and a much better estimate for the metallicity of the most metal-poor stars (currently $[\mathrm{Fe} / \mathrm{H}]=-4.0$ dex). A detailed study of those extremely rare $r$-process-enhanced stars that may be found-similar to that of Cayrel et al. (2001), will improve our age estimates for the Galactic halo based on radioactive decays of unstable heavy elements.

The entire Keck/HIRES user community owes a huge debt to Jerry Nelson, Gerry Smith, Steve Vogt, and many other people who have worked to make the Keck Telescope and HIRES a reality and to operate and maintain the Keck Observatory. We are grateful to the W. M. Keck Foundation for the vision to fund the construction of the W. M. Keck Observatory. The authors wish to extend special thanks to those of Hawai'ian ancestry on whose sacred mountain we are privileged to be guests. Without their generous hospitality, none of the observations presented herein would have been possible. This publication makes use of data products from the Two Micron All Sky Survey, which is a joint project of the University of Massachusetts and the Infrared Processing and Analysis Center/California Institute of Technology, funded by the National Aeronautics and Space Administration and the National Science Foundation. This research has made use of the SIMBAD database, operated at CDS, Strasbourg, France. We thank $\mathrm{T}$. Gehren and J. Reetz for providing us with Balmer line profiles and making the spectrum investigation utility SIU available to us. We thank the referee, Bruce Carney, for his thoughtful and constructive comments. We thank Sarah Yost, James Chakan, and Hubert Chen for obtaining the $B V$ photometry of BS 17447-0029. T. C. B acknowledges partial support for this work from grants AST 00-98508 and AST 00-98549 from the National Science Foundation. 
Arce, H. G., \& Goodman, A. A. 1999, ApJ, 512, L135

Allende Prieto, C., Asplund, M., García López, R. J., \& Lambert, D. L. 2002, ApJ, 567, 544

Allende Prieto, C., Barklem, P. S., Asplund, M., \& Ruiz Cobo, B. 2001, ApJ, 558, 830

Anthony-Twarog, B. J., Sarajedini, A., Twarog, B. A., \& Beers, T. C. 2000, AJ, 119, 2882

Alonso, A., Arribas, S., \& Martínez-Roger, C. 1994, A\&AS, 107, 365 1996, A\&A, 313, 873 1999, A\&AS, 140, 261 (erratum 376, 1039 [2001])

Arribas, S. \& Martínez-Roger, C. 1987, A\&AS, 70, 303

Asplund, M., Nordlund, A., Trampedach, R., Allende Prieto, C., \& Stein, R. F. 2000, A\&A, 359, 729

Bekki, K., \& Chiba, M. 2001, ApJ, 558, 666

Beers, T. C. 1999, in ASP Conf. Ser. 165, The Third Stromlo Symposium, ed. B. K. Gibson, T. S. Axelrod, \& M. E. Putman (San Francisco: ASP), 202

Springer), 3

Beers, T. C., et al. 2002, in preparation

Beers, T. C., Preston, G. W., \& Shectman, S. A. 1985, AJ, 90, 2089 1992, AJ, 103, 1987

Beers, T. C., Rossi, S., Norris, J. E., Ryan, S. G., \& Shefler, T. 1999, AJ, 117,981

Bergbusch, P. A., \& VandenBerg, D. A. 1992, ApJS, 81, 163

Bonifacio, P., Monai, S., \& Beers, T. C. 2000, AJ, 120, 2065

Carpenter, J. M. 2001, AJ, 121, 2851

Carretta, E., Gratton, R., Cohen, J. G., Beers, T. C., \& Christlieb, N. 2002,

AJ, 124, 481 (Paper II)

Cayrel, R., et al. 2001, Nature, 409, 691

Chiba, M., \& Beers, T. C. 2001, ApJ, 549, 325

Christlieb, N. 2000, Ph.D. thesis, Univ. Hamburg

Christlieb, N., \& Beers, T. C. 2000, in Proc. 2d Subaru HDS Workshop, ed. M. Takada-Hidai \& H. Ando (Tokyo: Natl. Astron. Obs. Japan), 255

Christlieb, N., et al. 2002, in preparation

Christlieb, N., Green, P. J., Wisotzki, L., \& Reimers, D. 2001a, A\&A, 375, 366

Christlieb, N., Wisotzki, L., Reimers, D., Homeier, D., Koester, D., \& Heber, U. 2001b, A\&A, 366, 898

Cohen, J. G., Behr, B. B., \& Briley, M. M. 2001, AJ, 122, 1420

Cohen, J. G., Frogel, J. A.. Persson, S. E. \& Elias, J. H. 1981, ApJ, 249, 481

Dalle Ore, C. M. 1992, Ph.D. thesis, Univ. California, Santa Cruz

de Bernardis, P., et al. 2000, Nature, 404, 955

Depagne, E., Hill, V., Christlieb, N., \& Primas, F. 2000, A\&A, 364, L6

Dickey, J. M., \& Lockman, F. J. 1990, ARA\&A, 28, 215

Edvardsson, B., Gustafsson, B., Johansson, S. G., Kiselman, D., Lambert,

D. L., Nissen, P. E., \& Gilmore, G. 1994, A\&A, 290, 176

Fulbright, J. P. 2000, AJ, 120, 1841

Fuhrmann, K. 1998, A\&A, 338, 161

Fuhrmann, K., Axer, M., \& Gehren, T. 1993, A\&A, 271, 451

Gezari, D. Y., Pitts, P. S., \& Schmitz, W. 1999, Catalog of Infrared Observations (version 5.1; Greenbelt, MD: GSFC)
Gratton, R., et al. 2002, in preparation
Gratton, R. G., Carretta, E., \& Castelli, F. 1996, A\&A, 314, 191

Gratton, R. G., Carretta, E., Eriksson, K., \& Gustafsson, B. 1999, A\&A, 350,955

Gustafsson, B., Bell, R. A., Eriksson, K., \& Nordlund, A. 1975, A\&A, 42, 407

Houdashelt, M. L., Bell, R. A., \& Sweigart, A. V. 2000, AJ, 119, 1448

Ivans, I. I., Kraft, R. P., Sneden, C., Smith, G. H., Rich, R. M., \& Shetrone, M. 2001, AJ, 122, 1438

Korn, A. J., \& Gehren, T. 2001, in ASP Conf. Ser. 228, Dynamics of Star Clusters and the Milky Way, ed. S. Deiters, B. Fuchs, A. Just, R. Spurzem, \& R. Wielen (San Francisco: ASP), 494

Kurucz, R. L. 1993, CD-ROM 13, ATLAS9 Stellar Atmosphere Programs and $2 \mathrm{~km} / \mathrm{s}$ Grid (Cambridge: Smithsonian Astrophys. Obs.)

2002, in The Link between Stars and Cosmology, ed. M. Chávez, A. Bressan, A. Buzzoni, \& D. Mayya (Dordrecht: Kluwer), in press

Laird, J. B., Carney, B. W., \& Latham, D. W. 1988, AJ, 95, 1843

Lebreton, Y. 2000, ARA\&A, 38, 35

McCarthy, J. K. 1988, Ph.D. thesis, Caltech

McWilliam, A. 1997, ARA\&A, 35, 503

McWilliam, A., Preston, G. W., Sneden, C., \& Searle, L. 1995, AJ, 109, 2757

Mermilliod, J.-C., Mermilliod, M., \& Hauck, B. 1997, A\&AS, 124, 349

Nikolaev, S., Weinberg, M. D., Skrutskie, M. F., Cutri, R. M., Wheelock, S. L., Gizis, J. E., \& Howard, E. M. 2000, AJ, 120, 3340

Nissen, P. E., Gustafsson, B., Edvardsson, B., \& Gilmore, G. 1994, A\&A, 285,440

Norris, J. E., Beers, T. C., \& Ryan, S. G. 2000, ApJ, 540, 456

Norris, J. E., Ryan, S. G., \& Beers, T. C. 2001, ApJ, 561, 1034

Norris, J. E., Ryan, S. G., Beers, T. C., \& Deliyannis, C. P. 1997, ApJ, 485 , 370

Preston, G. W., Shectman, S. A., \& Beers, T. C. 1991, ApJS, 76, 1001

Ramírez, S. V., Cohen, J. G., Buss, J., \& Briley, M. M. 2001, AJ, 122, 1429

Ryan, S. G., Norris, J. E., \& Beers, T. C. 1996, ApJ, 471, 254 1999, ApJ, 523, 654

Ryan, S. G., Norris, J. E. \& Bessell, M. S. 1991, AJ, 102, 303

Schlegel, D. J., Finkbeiner, D. P. \& Davis, M. 1998, ApJ, 500, 525

Shortridge, K. 1993, in ASP Conf. Ser. 52, Astronomical Data Analysis Software and Systems II, ed. R. J. Hanisch, R. J. V. Brissenden, \& J. Barnes (San Francisco: ASP), 219

Skrutskie, M. F., et al. 1997, in The Impact of Large Scale Near-IR Sky Surveys, ed. F. Garzón, N. Epchtein, A. Omont, W. B. Burton, \& P. Pers (Dordrecht: Kluwer), 25

Thévenin, F., \& Idiart, T. P. 1999, ApJ, 521, 753

Vogt, S. E., et al. 1994, Proc. SPIE, 2198, 362

Wisotzki, L., Christlieb, N., Bade, N., Beckmann, V., Köhler, T., Vanelle, C., \& Reimers, D. 2000, A\&A, 358, 77

Wisotzki, L., Köhler, T. Groote, D. \& Reimers, D. 1996, A\&AS, 115, 227

Yi, S., Demarque, P., Kim, Y.-C., Lee, Y.-W., Ree, C. H., Lejeune, T., \& Barnes, S. 2001, ApJS, 136, 417

Zacharias, N., et al. 2000, AJ, 120, 2131 